Article

\title{
Powdery Mildew Resistance Phenotypes of Wheat Gene Bank Accessions
}

\author{
Antonín Dreiseitl (iD
}

Citation: Dreiseitl, A. Powdery Mildew Resistance Phenotypes of Wheat Gene Bank Accessions. Biology 2021, 10, 846. https://doi.org/ 10.3390/biology10090846

Academic Editors: Laura Pascual,

Rosa Mérida-García

Eugene Metakovsky and

Robert Henry

Received: 25 June 2021

Accepted: 20 August 2021

Published: 30 August 2021

Publisher's Note: MDPI stays neutral with regard to jurisdictional claims in published maps and institutional affiliations.

Copyright: (C) 2021 by the author. Licensee MDPI, Basel, Switzerland. This article is an open access article distributed under the terms and conditions of the Creative Commons Attribution (CC BY) license (https:/ / creativecommons.org/licenses/by/ $4.0 /)$.
Department of Integrated Plant Protection, Agrotest Fyto Ltd., Havlíčkova 2787, CZ-767 01 Kroměříž, Czech Republic; dreiseitl@vukrom.cz; Tel.: +420-573-317-139

Simple Summary: Bread wheat is one of the most important sources of human and animal food and powdery mildew is a serious disease of this crop. Breeding and growing resistant cultivars are an effective and environmentally friendly way of reducing the adverse impact of the disease on grain yield and quality. The main aim of this study was to detect major resistances against powdery mildew in a set of wheat accessions from the Czech gene bank and to group them according to their responses. Ear progenies of 448 varieties originating from 33 countries were inoculated with three isolates of the pathogen. One hundred and ten varieties showed resistance to at least one isolate and 59 varieties were resistant to all three isolates. Resistance to the three isolates was present mostly in varieties of Northwest Europe and was more than three times more frequent in spring than in winter wheats. Results will facilitate a rational and practical approach of breeding new wheat cultivars using this set of gene bank accessions as recipients of novel genes from wheat-related species and accumulate minor resistance genes to improve resistance durability.

Abstract: Powdery mildew (Blumeria graminis f. sp. tritici) is a common pathogen of bread wheat (Triticum aestivum L.), and genetic resistance is an effective and environmentally friendly method to reduce its adverse impact. The introgression of novel genes from wheat progenitors and related species can increase the diversity of disease resistance and accumulation of minor genes to improve the crop's resistance durability. To accomplish these two actions, host genotypes without major resistances should be preferably used. Therefore, the main aim of this study was to carry out seedling tests to detect such resistances in a set of wheat accessions from the Czech gene bank and to group the cultivars according to their phenotype. Ear progenies of 448 selected cultivars originating from 33 countries were inoculated with three isolates of the pathogen. Twenty-eight cultivars were heterogeneous, and 110 cultivars showed resistance to at least one isolate. Fifty-nine cultivars, mostly from Northwest Europe, were resistant to all three isolates were more than three times more frequently recorded in spring than in winter cultivars. Results will facilitate a rational and practical approach preferably using the set of cultivars without major resistances for both mentioned methods of breeding wheat cultivars resistant to powdery mildew.

Keywords: Blumeria graminis f. sp. hordei; Blumeria graminis f. sp. tritici; infection response arrays; resistance postulation; single ear progenies

\section{Introduction}

Plant diseases cause substantial losses in crop production and compromise food safety due to the presence of pesticides and toxins [1]. Bread wheat (Triticum aestivum L.) is one of the most important sources of human and animal food. Powdery mildew, caused by the biotrophic airborne fungal pathogen Blumeria graminis f. sp. tritici $(B g t)$ is a serious disease of wheat in most parts of the world that reduces yield and quality [2]. Breeding and growing resistant cultivars are an effective and environmentally friendly way of reducing the adverse impact of mildew. However, as is the case with barley mildew (Blumeria graminis f. sp. hordei $=B g h$ ) [3], the use of race-specific resistance in wheat is not durable 
because evolutionary forces operating on cereal mildews result in extremely high diversity and adaptability of their populations $[4,5]$. The transfer of resistances derived from wild relatives of bread wheat could be a more effective method of disease management. Over the last decades, technologies connected with breeding have made significant strides and the knowledge gained is accelerating the identification of key resistance traits that can be efficiently transferred and applied to crop breeding programs [6].

Bread wheat is a hexaploid species that has evolved in the last $0.3-0.5$ million years by spontaneous hybridization of originally diploid species and consists of three subgenomes designated as A, B, and D. The A subgenome was contributed by wild einkorn wheat $T$. urartu, the B subgenome by an unknown species closely related to Aegilops speltoides, and the D subgenome originated from A. tauschii [7]. Wheat is thus related to a range of species belonging to its primary, secondary and tertiary gene pools [8]. Introgression of novel genes from wheat progenitors and related species can increase the diversity of agronomically important traits such as disease resistance, which are invaluable in the breeding of the crop.

Research presented in this contribution is a prerequisite for a project of wheat genomewide association study (GWAS) involved with a large-scale analysis of correlations between phenotypes of many accessions and aimed to identify genes associated with drought and frost tolerance, resistance to ear fusariosis and genes affecting developmental stages of plants and especially flowering time.

The goal of this study was to define resistance of potential genotypes of wheat, i) as recipients for introgressing powdery mildew resistance derived from Triticum militinae [9] and T. monococcum [10], and ii) for accumulating minor resistance genes from the tested cultivars. The aim of the current tests was to detect resistances based on major genes to powdery mildew at the seedling stage and to group the cultivars according to resistance phenotype.

\section{Materials and Methods}

The following methods, especially in Sections 2.2 and 2.3, are similar to those previously described [11].

\subsection{Plant Material and Pathogen Isolates}

Ear progenies of 448 cultivars selected from the Czech wheat gene bank and multiplied in rows in the field were studied. For seedling resistance tests two domestic (Czech) and one Russian isolate of $B g t$ were used. Isolate E originated from a wheat field between Dačice and Chlumec in May 2018, isolate Tm-258 was collected from an experimental line Tm-258 in Olomouc in May 2011, and isolate Galina was recovered from a cultivar of the same name in the St. Petersburg area in July 2018. Their virulence/avirulence patterns were determined on eight selected wheat cultivars (see later). The isolates were multiplied on leaf segments of susceptible winter wheat AF Jumiko and fresh conidia were used for inoculation.

\subsection{Testing Procedure}

For in vitro resistance tests about five seeds of each accession were sown in pots ( $80 \mathrm{~mm}$ diameter) containing a gardening peat substrate and placed in a mildew-proof greenhouse under natural daylight. The primary leaves were excised when the second leaves were emerging, and leaf segments $20 \mathrm{~mm}$ long were cut from the middle part of healthy fully expanded leaves. Five segments of each accession were deposited on the surface of media $\left(0.8 \%\right.$ water agar containing $40 \mathrm{mg}^{-\mathrm{L}}$ of benzimidazole-a leaf senescence inhibitor) in a $150 \mathrm{~mm}$ Petri dish. Leaf segments were placed next to each other with their adaxial surfaces facing upward.

For inoculation, a cylindrical metal settling tower of $150 \mathrm{~mm}$ diameter and $415 \mathrm{~mm}$ in height was used and a dish with leaf segments was put at the bottom of the tower. Conidia of each isolate, taken from leaf segments of the susceptible cultivar with fully developed pathogen colonies, were shaken onto a square piece $(40 \times 40 \mathrm{~mm})$ of black paper to visually control the amount of inoculum deposited. Then, the paper was rolled 
to form a blowpipe, and conidia of the isolate were blown through a side hole of $13 \mathrm{~mm}$ diameter in the upper part of the settling tower over the Petri dish at a concentration of ca. 20 conidia $\mathrm{mm}^{-2}$. Before inoculation with another isolate the settling tower and other tools were sterilised with ethyl-alcohol $96 \%$. The dishes with inoculated leaf segments were incubated at $18 \pm 1{ }^{\circ} \mathrm{C}$ under artificial light (cool-white fluorescent lamps providing $12 \mathrm{~h}$ light at $30 \pm 5 \mu \mathrm{mol} \mathrm{m}{ }^{-2} \mathrm{~s}^{-1}$ ).

\subsection{Evaluation}

Seven days after inoculation, infection response (IR = phenotype of accession $\mathrm{x}$ isolate interaction) on the adaxial side of leaf segments (Figure 1) were scored on a scale $0-4$, where $0=$ no mycelium and sporulation, and $4=$ strong mycelial growth and sporulation [12] IRs 3, 3-4 and 4 were considered susceptible. Each cultivar was tested once with the three isolates and subsequently with a colony isolate derived from each of the three isolates. If there were significant differences in IRs between them, additional tests were done.

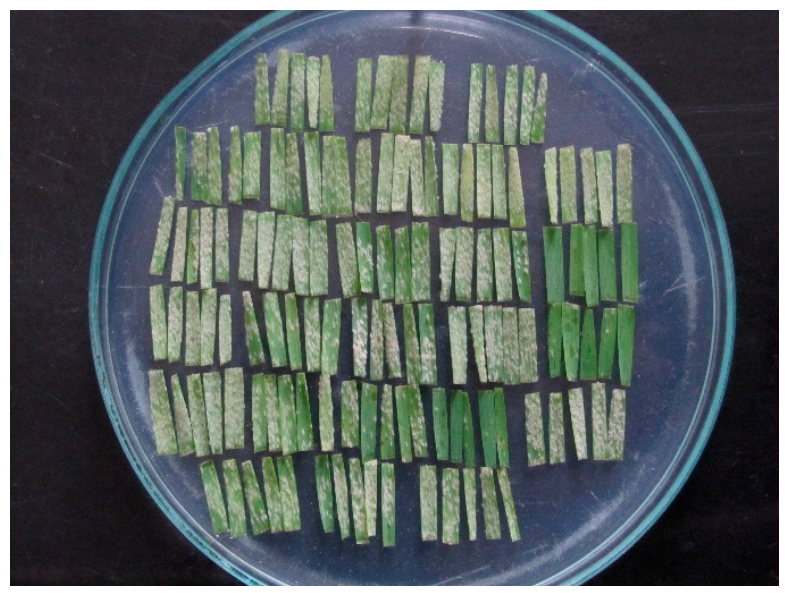

Figure 1. Twenty-six wheat cultivars each represented with a pentad of leaf segments seven days after inoculation with a Blumeria graminis f. sp. tritici isolate.

\subsection{Numerical Designation of Resistance Groups}

Resistance phenotypes characterised by IRs of a cultivar to the three $B g h$ isolates formed an infection response array (IRA) used for numerical designation of its resistance group. If there was a resistant response to a corresponding isolate, the first isolate was given the value $1\left(2^{0}\right)$, the second isolate $=2\left(2^{1}\right)$, and the third isolate $4\left(2^{2}\right)$. Therefore, a digit can have a value from 0 (no resistance to any of the three isolates) up to $7(=1+2+4)$ denoting resistance to each of the three isolates. The resulting number (reverse-octal) defines phenotypic classification of the resistance/susceptibility pattern of each cultivar and its resistance group.

\section{Results}

Four hundred and forty-eight wheat cultivars were tested, of which 422 were winter and 26 spring growth habit; 420 cultivars were homogeneous whereas 28 showed heterogeneous IRAs when two or more IRs were detected in one or more cultivar-isolate interactions (Table 1).

Major resistance to powdery mildew was found in 110 homogeneous accessions, 95 of which were resistant to the Tm-258 isolate, 71 resistant to the isolate $\mathrm{E}$ and 92 cultivars to the Galina isolate. According to their responses to the isolates, homogeneous cultivars were divided into eight groups (Table 2). Twenty-one cultivars were resistant to one isolate (sum of groups 1, 2 and 4), 30 were resistant to two isolates (groups 3, 5 and 6) and there was resistance to all three isolates in 59 cultivars (group 7). Susceptibility to all isolates was the most frequent, detected in 310 cultivars (group 0). 
Table 1. Four hundred and forty-eight wheat cultivars, their growth type, country of origin and response to three powdery mildew isolates coded in reverse-octal notation (resistance group).

\begin{tabular}{|c|c|c|c|c|c|c|c|c|}
\hline Cultivar $^{1}$ & $\mathrm{O}^{2}$ & $\mathrm{G}^{3}$ & Cultivar & $\mathrm{O}$ & $\mathrm{G}$ & Cultivar & $\mathrm{O}$ & G \\
\hline Activus & 2 & 5 & Farabi & 20 & 0 & Norstar & 6 & 0 \\
\hline Addict & 13 & 7 & Faunus & 2 & 5 & Novosibirskaya 2 & 27 & 6 \\
\hline AF Jumiko & 8 & 0 & Faustus & 10 & 7 & Novosibirskaya 3 & 27 & 0 \\
\hline Airbus & 13 & 0 & Federer & 8 & 0 & Novosibirskaya 32 & 27 & 0 \\
\hline Akasabishirazu 1 & 19 & 0 & Fenomen & 13 & 7 & Novosibirskaya 40 & 27 & 0 \\
\hline Akteur & 10 & 0 & Feria & 13 & 0 & Odesskaja 16 & 32 & 0 \\
\hline Aladin & 10 & 0 & Fermi & 13 & 7 & Odesskaja 66 & 32 & 0 \\
\hline Alana & 8 & 0 & Filon & 13 & 4 & Odeta & 8 & 7 \\
\hline Albertus & 2 & 7 & Fisht & 27 & 0 & Oska & 8 & 0 \\
\hline Alceste & 24 & 7 & Florett & 13 & 7 & Pajbjerg 184 & 11 & 0 \\
\hline Alexander & 10 & 0 & Florian & 10 & 0 & Pankratz & 10 & 0 \\
\hline Alibaba & 10 & 0 & Forhand & 8 & 0 & Pannonia NS & 28 & 0 \\
\hline Alicia & 8 & 5 & Franz & 10 & 7 & Papageno & 2 & 7 \\
\hline Aliya & 20 & 0 & Frisky & 13 & 4 & Partner & 13 & 7 \\
\hline Alka & 8 & 0 & Gallio & 2 & 5 & Patras & 10 & 0 \\
\hline Alomar & 34 & 7 & Gaudio & 10 & 5 & Penalta & 8 & 0 \\
\hline Alpine Neuzucht & 2 & 0 & Genius & 10 & 0 & Penelope & 8 & 0 \\
\hline Altigo & 13 & $\mathrm{~h}$ & Globus & 10 & 0 & Petrus & 10 & 0 \\
\hline Amandus & 2 & 0 & Gordian & 17 & 5 & Pexeso & 8 & 1 \\
\hline Anara & 20 & $\mathrm{~h}$ & Gourmet & 34 & 5 & Pilgrim PZO & 10 & 7 \\
\hline Andrejka & 8 & 0 & Grafton & 14 & 0 & Pionier & 10 & 0 \\
\hline Angelus & 2 & 0 & Graindor & 13 & 0 & Pitbull & 10 & 7 \\
\hline Aniya & 20 & $\mathrm{~h}$ & Grana & 25 & 0 & Plantahof 3 & 17 & 0 \\
\hline Annie & 8 & 0 & Granny & 8 & 5 & Ponticus & 10 & 7 \\
\hline Antonius & 2 & 0 & Grizzly & 8 & 0 & Porthus & 10 & 7 \\
\hline Apache & 13 & 0 & Hadmerslebener Q. & 9 & 0 & Postoloprtska Pres. & 7 & 0 \\
\hline Apanage & 13 & 4 & Hana & 8 & 0 & Potenzial & 10 & 0 \\
\hline Apertus & 10 & 0 & Hanacka Osinata & 8 & 0 & Praskoviy & 27 & 0 \\
\hline Apollo & 24 & 0 & Hanswin & 17 & 0 & Preciosa & 24 & 0 \\
\hline Apostel & 10 & 7 & Hedvika & 24 & 0 & Premio & 13 & 0 \\
\hline Arina & 17 & 0 & Henrik & 13 & 0 & Prestizh & 27 & $\mathrm{~h}$ \\
\hline Arkadia & 25 & 0 & Hermann & 13 & 0 & Prince Leopold & 3 & 1 \\
\hline Arkeos & 13 & 0 & Hewitt & 24 & 7 & Princeps & 10 & 0 \\
\hline Arktis & 10 & 6 & Hondia & 25 & 0 & Proteus & 13 & 0 \\
\hline Artist & 10 & 0 & Chevalier & 10 & 0 & Pyselka & 8 & 0 \\
\hline Asta & 8 & 0 & Chevignon & 13 & 7 & Quintus & 8 & 5 \\
\hline Astella & 7 & 0 & Chiron & 10 & $\mathrm{~h}$ & Raduza & 8 & 0 \\
\hline Astet & 32 & 0 & Chlumecka 12 & 8 & 0 & Rapsodia & 14 & 0 \\
\hline Astrid & 8 & 5 & Ibarra & 8 & 0 & Rassad & 20 & 0 \\
\hline Athlon & 13 & 7 & Illusion & 8 & $\mathrm{~h}$ & Rebell & 10 & 0 \\
\hline Atlas 66 & 33 & 0 & Immendorfer Kolben & 2 & 0 & Regina & 8 & 1 \\
\hline Atomic & 10 & 0 & Inspiration & 10 & 0 & Registana & 8 & 5 \\
\hline Attraktion & 10 & 7 & Iron & 30 & 0 & Renan & 13 & 0 \\
\hline Atuan & 10 & 7 & IS Agape & 29 & 0 & Rexia & 8 & 0 \\
\hline Avenue & 13 & 5 & IS Conditor & 29 & 0 & RGT Cesario & 13 & 0 \\
\hline Axioma & 10 & 0 & IS Danubius & 29 & 0 & RGT Matahari & 8 & 0 \\
\hline Bagou & 13 & 7 & IS Escoria & 29 & 0 & RGT Mobidick & 8 & 0 \\
\hline Bakfis & 8 & 0 & IS Gordius & 29 & 0 & RGT Premiant & 8 & 0 \\
\hline Balan de Figanesti & 26 & 0 & IS Jarissa & 29 & 7 & RGT Reform & 10 & $\mathrm{~h}$ \\
\hline Baletka & 8 & 0 & IS Laudis & 29 & 1 & RGT Sacramento & 13 & 0 \\
\hline Balitus & 2 & 5 & Ivanovskaja 12 & 32 & 0 & Rheia & 8 & 0 \\
\hline Bamberka & 25 & 0 & Izalco CS & 13 & 0 & Rivero & 10 & 7 \\
\hline Banderola & 25 & 0 & $I z z y$ & 8 & 7 & Rockefeller & 10 & 7 \\
\hline Bankuta & 16 & 0 & Jensen & 11 & 0 & Rodnik Tarasovskij & 27 & 0 \\
\hline Bankuti 8000 & 16 & 0 & Jindra & 8 & 2 & Ronsard & 13 & 6 \\
\hline Banquet & 8 & 0 & Johnson & 13 & 7 & Rosatch & 17 & 0 \\
\hline Barabas Fele & 16 & 0 & Jubile II & 3 & 0 & Rumor & 10 & 0 \\
\hline Baracuda & 10 & 7 & Judita & 8 & 0 & Rumunka & 5 & 0 \\
\hline Barbarossa Podol & 25 & 0 & Julie & 8 & 0 & Rusalka & 4 & 0 \\
\hline Bardan & 17 & 0 & Julius & 10 & 0 & Rytmus & 8 & 0 \\
\hline Bardotka & 8 & 0 & Juna & 8 & $\mathrm{~h}$ & Safari & 10 & 0 \\
\hline Barroko & 14 & $\mathrm{~h}$ & Kabot & 10 & 5 & Sailor & 10 & 7 \\
\hline Barryton & 13 & 0 & Kanhard Sel. Buck & 1 & 0 & Sakura & 8 & 0 \\
\hline Basilio & 13 & 0 & Kanzler & 10 & 0 & Sally & 8 & 0 \\
\hline Batis & 10 & $\mathrm{~h}$ & Kasticka Osinatka & 8 & 0 & Samanta & 8 & 0 \\
\hline Batkan Krasnaya & 21 & 0 & Kelvin & 24 & $\mathrm{~h}$ & Samara & 8 & 0 \\
\hline
\end{tabular}


Table 1. Cont.

\begin{tabular}{|c|c|c|c|c|c|c|c|c|}
\hline Cultivar ${ }^{1}$ & $\mathrm{O}^{2}$ & $\mathrm{G}^{3}$ & Cultivar & $\mathrm{O}$ & $\mathrm{G}$ & Cultivar & $\mathrm{O}$ & G \\
\hline BC Anica & 15 & $\mathrm{~h}$ & Kitri & 8 & 7 & Samurai & 10 & 0 \\
\hline BC Darija & 15 & 5 & Kodex & 10 & 0 & San Pastore & 18 & 0 \\
\hline BC Lira & 15 & 0 & Kometus & 10 & 5 & Sarka & 8 & 0 \\
\hline Beduin & 13 & 0 & Kompass & 10 & 0 & Sarmund & 10 & 0 \\
\hline Bekend & 8 & 7 & Korneuburger & 2 & 0 & Saskia & 8 & 0 \\
\hline Belgrade 1 & 28 & 0 & Korneuburger Gran. & 2 & 0 & Saxo & 30 & 0 \\
\hline Benschmark & 11 & 7 & Kosutka & 8 & 0 & Seance & 8 & 5 \\
\hline Bermude & 13 & 0 & Kredo & 10 & 7 & Secese & 8 & 0 \\
\hline Bernstein & 10 & 1 & Kulundinka & 27 & 0 & Seladon & 8 & 0 \\
\hline Bezostaja 1 & 27 & 0 & Kurt & 10 & 7 & Sepia & 13 & 4 \\
\hline Bienfait & 13 & 0 & KWS Dacanto & 10 & 0 & Sepstra & 10 & 0 \\
\hline Biscay & 10 & 0 & KWS Emil & 10 & 5 & Septima & 8 & 5 \\
\hline Bizel & 13 & 0 & KWS Eternity & 10 & 0 & Seu Seun 8 & 22 & 0 \\
\hline Bodycek & 8 & 0 & KWS Ferrum & 10 & 0 & Sheriff & 11 & $\mathrm{~h}$ \\
\hline Bohemia & 8 & 0 & KWS Fontas & 10 & 0 & Sida & 8 & 0 \\
\hline Boisseau & 13 & 7 & KWS Loft & 10 & 7 & Sila & 27 & 0 \\
\hline Bonanza & 10 & 7 & KWS Magic & 10 & 0 & Silvanus & 29 & 0 \\
\hline Boregar & 13 & 0 & KWS Mairra & 10 & 7 & Simila & 8 & 2 \\
\hline Botagoz & 20 & 0 & KWS Montana & 10 & 0 & Siria & 7 & 0 \\
\hline Brea & 8 & 0 & KWS Ronin & 10 & 0 & Skorpion & 8 & 0 \\
\hline Brentano & 10 & $\mathrm{~h}$ & KWS Santiago & 14 & 7 & Slovenska 777 & 8 & 0 \\
\hline Brigala & 27 & 0 & KWS Silverstone & 14 & 0 & Smaragd & 10 & 0 \\
\hline Brilliant & 10 & 0 & KWS Smart & 10 & 0 & Sofolk & 13 & 2 \\
\hline Brokat & 10 & 0 & Landsknecht & 10 & 0 & Sofru & 13 & 0 \\
\hline Buteo & 10 & 0 & Laurier & 13 & $\mathrm{~h}$ & Solindo & 13 & 7 \\
\hline Butterfly & 8 & 0 & Lavantus & 10 & 0 & Somtuoso CS & 13 & 0 \\
\hline Calisol & 13 & 0 & Lavoiser & 13 & $\mathrm{~h}$ & Sonergy & 13 & 0 \\
\hline Calumet & 13 & 0 & Lear & 14 & 0 & Sosthene & 13 & 0 \\
\hline Caphorn & 14 & 1 & Legenda Mironovsk. & 32 & $\mathrm{~h}$ & Sparta & 8 & 0 \\
\hline Capone & 10 & 0 & Leguan & 8 & 5 & Spontan & 10 & $\mathrm{~h}$ \\
\hline Carmina & 8 & 0 & Lemaire 4 & 13 & 0 & Stadium & 13 & 7 \\
\hline Cecilius & 2 & 0 & Lena & 8 & $\mathrm{~h}$ & Steffi & 8 & $\mathrm{~h}$ \\
\hline Cellule & 13 & 0 & Lennox & 10 & 5 & Stupicka Bastard & 8 & 0 \\
\hline Ceska Presivka & 7 & 0 & LG Imposanto & 10 & 0 & SU Kae no. 169 & 22 & 7 \\
\hline Ceylon & 12 & 0 & LG Magirus & 10 & 0 & Sulamit & 8 & 2 \\
\hline Cimrmanova Rana & 8 & 0 & LG Mocca & 10 & 7 & Sultan & 8 & 0 \\
\hline Citrus & 10 & 0 & Libertina & 8 & 5 & Sumai 3 & 19 & 0 \\
\hline Clever & 14 & 0 & Litera & 26 & 0 & Svitava & 8 & 0 \\
\hline Cocoon & 13 & 0 & Lithium & 13 & $\mathrm{~h}$ & SW Kadrilij & 30 & 7 \\
\hline Collector & 13 & 0 & Loosedorfer Winter. & 2 & 0 & SY Alteo & 13 & 0 \\
\hline Colonia & 10 & 7 & Lorien & 8 & 0 & SY Mattis & 13 & 0 \\
\hline Complet & 10 & 0 & Lotte & 8 & $\mathrm{~h}$ & SY Passport & 10 & 7 \\
\hline Complice & 13 & 0 & Lovaszpatonai 157 & 16 & 0 & Tabasco & 10 & 7 \\
\hline Conexion & 13 & 0 & Lovrin 13 & 26 & 0 & Tarasovskaya Ostist. & 27 & 0 \\
\hline Corsaire & 13 & 0 & Ludwig & 2 & 0 & Tau & 27 & 0 \\
\hline Coutiches & 13 & 0 & Lukullus & 2 & 5 & Tercie & 8 & 5 \\
\hline Cubus & 10 & 1 & Luna & 25 & 0 & Terroir & 13 & 0 \\
\hline Dafne & 8 & 7 & Magister & 10 & 0 & Tervel & 4 & 0 \\
\hline Dagmar & 8 & 0 & Magnifik & 12 & 3 & Tiguan & 13 & 0 \\
\hline Dalmatia 2 & 15 & 0 & Magno & 17 & 0 & Tilman & 13 & 7 \\
\hline Dancing Queen & 8 & 7 & Maira & 20 & 0 & Timing & 13 & 4 \\
\hline Dankowska Biala & 25 & 0 & Manitou & 10 & 7 & Tir & 31 & 0 \\
\hline Darwin & 10 & 0 & Mara & 8 & 0 & Tobak & 10 & 0 \\
\hline Diadem & 8 & 0 & Marquardt II & 10 & 0 & Todireshti & 23 & 0 \\
\hline Dichter & 10 & 0 & Master's New Y. & 14 & 0 & Tonnage & 11 & 7 \\
\hline Dmitriy & 27 & 0 & Matchball & 8 & 0 & Torp & 11 & 5 \\
\hline Drifter & 10 & 0 & Matylda & 8 & 0 & Tosca & 8 & 0 \\
\hline Dromos & 10 & 0 & Meritto & 8 & 0 & Tower & 24 & 0 \\
\hline Duecentodieci & 18 & 0 & Mescal & 10 & 0 & Trappe & 10 & 0 \\
\hline Dulina & 8 & 0 & Messi & 8 & 0 & Trumf (Heines IV) & 10 & 0 \\
\hline Ebi & 10 & 0 & Midas & 2 & 0 & Tuerkis & 10 & 0 \\
\hline Edgar & 10 & 0 & Minhardi & 33 & 0 & Tulecka & 25 & 0 \\
\hline Elan & 10 & 0 & Miranda & 26 & 0 & Turandot & 8 & 0 \\
\hline Elixer & 13 & $\mathrm{~h}$ & Mironovska & 8 & $\mathrm{~h}$ & Tvorec & 27 & 0 \\
\hline Elly & 8 & 0 & Mladka & 8 & 0 & Tybalt & 10 & 7 \\
\hline Emilio & 2 & 0 & Mona & 8 & 0 & Uljanovka & 27 & 2 \\
\hline Energo & 2 & 0 & Montaldo & 17 & 0 & Urup & 27 & $\mathrm{~h}$ \\
\hline Epi d_Or & 13 & 7 & Mozes & 10 & 1 & Valticka Osinata B & 7 & 0 \\
\hline
\end{tabular}


Table 1. Cont.

\begin{tabular}{|c|c|c|c|c|c|c|c|c|}
\hline Cultivar ${ }^{1}$ & $\mathrm{O}^{2}$ & $\mathrm{G}^{3}$ & Cultivar & $\mathrm{O}$ & G & Cultivar & $\mathrm{O}$ & G \\
\hline Epos & 10 & 7 & Mulan & 10 & 0 & Vanek & 8 & $\mathrm{~h}$ \\
\hline Eroica & 30 & 0 & Mutic & 13 & 0 & Vanessa & 8 & 0 \\
\hline Ershovskaya 10 & 27 & 0 & MV Beres & 16 & 0 & Venistar & 29 & 0 \\
\hline Estevan & 2 & 0 & MV Bodri & 16 & 0 & Viki & 8 & 0 \\
\hline Estica & 24 & 0 & MV Kolompos & 16 & 0 & Viriato & 13 & 2 \\
\hline Estivus & 10 & 0 & MV Nador & 16 & 0 & Vlasta & 8 & 0 \\
\hline Etana & 10 & 0 & MV Nemere & 16 & $\mathrm{~h}$ & Volodarka & 32 & 0 \\
\hline Etela & 8 & 0 & MV Pengo & 16 & 0 & Vouska z Tremos. & 8 & 0 \\
\hline Etuos & 10 & 7 & MV Zelma & 16 & 3 & Weibulls Trond & 30 & 0 \\
\hline Euclide & 13 & 0 & Nakskov & 11 & 0 & WPB Calgary & 24 & 7 \\
\hline Eurofit & 2 & 0 & Nelson & 10 & $\mathrm{~h}$ & Zdar & 8 & 1 \\
\hline Event & 10 & 0 & Nikol & 8 & 0 & Zeppelin & 10 & 0 \\
\hline Evina & 13 & 0 & Nordika & 8 & 0 & Zidlochovicka Osin. & 7 & 2 \\
\hline Fabius & 2 & 0 & Nordkap & 10 & 7 & Zora & 8 & 0 \\
\hline Fairway & 13 & 0 & Norin & 10 & 0 & - & - & - \\
\hline Fakir & 10 & 0 & Norin 40 & 19 & 0 & - & - & - \\
\hline
\end{tabular}

${ }^{1}$ Spring wheats are written in italics. ${ }^{2}$ Country of origin: 1 ARG Argentina, 2 AUT Austria, 3 BEL Belgium, 4 BGR Bulgaria, 5 BIH Bosnia and Herzegovina, 6 CAN Canada, 7 CSK Czechoslovakia, 8 CZE Czech Republic, 9 DDR German Democratic Republic, 10 DEU Germany, 11 DNK Denmark, 12 FIN Finland, 13 FRA France, 14 GBR Great Britain, 15 HRV Croatia, 16 HUN Hungary, 17 CHE Switzerland, 18 ITA Italy, 19 JPN Japan, 20 KAZ Kazakhstan, 21 KGZ Kyrgyzstan, 22 KOR Korea, 23 MDA Moldavia, 24 NLD Netherlands, 25 POL Poland, 26 ROM Romania, 27 RUS Russia, 28 SRB Serbia, 29 SVK Slovakia, 30 SWE Sweden, 31 TUR Turkey, 32 UKR Ukraine, 33 USA United States of America, 34 Unknown. ${ }^{3}$ Resistance group $(0-7), \mathrm{h}=$ heterogeneous.

Table 2. Infection response arrays (IRAs) of 420 homogeneous wheats represented by eight model cultivars separately inoculated with three isolates of powdery mildew and octal notation of infection responses to determine their resistance group.

\begin{tabular}{|c|c|c|c|c|c|}
\hline \multirow{2}{*}{$\begin{array}{l}\text { Model Wheat } \\
\text { Cultivar }\end{array}$} & \multicolumn{3}{|c|}{ Powdery Mildew Isolates } & \multirow{2}{*}{$\begin{array}{l}\text { Octal Notation } \\
\text { (Group) }\end{array}$} & \multirow{2}{*}{$\begin{array}{c}\text { Group } \\
\text { Frequency }(n)\end{array}$} \\
\hline & $\begin{array}{l}\text { Tm-258 } \\
\left(2^{0}=1\right)\end{array}$ & $\begin{array}{c}E \\
\left(2^{1}=2\right)\end{array}$ & $\begin{array}{l}\text { Galina } \\
\left(2^{2}=4\right)\end{array}$ & & \\
\hline AF Jumiko & $\mathrm{s}$ & $\mathrm{s}$ & $\mathrm{s}$ & 0 & 310 \\
\hline Pexeso & $\mathrm{r}$ & $\mathrm{s}$ & $\mathrm{s}$ & 1 & 9 \\
\hline Sulamit & $\mathrm{s}$ & $\mathrm{r}$ & $\mathrm{s}$ & 2 & 7 \\
\hline Magnifik & $\mathrm{r}$ & $\mathrm{r}$ & $\mathrm{s}$ & 3 & 2 \\
\hline Apanage & $\mathrm{s}$ & $\mathrm{s}$ & $\mathrm{r}$ & 4 & 5 \\
\hline Gourmet & $\mathrm{r}$ & $\mathrm{s}$ & $\mathrm{r}$ & 5 & 25 \\
\hline Ronsard & $\mathrm{s}$ & $\mathrm{r}$ & $\mathrm{r}$ & 6 & 3 \\
\hline Dancing Queen & $\mathrm{r}$ & $\mathrm{r}$ & $\mathrm{r}$ & 7 & 59 \\
\hline
\end{tabular}

Cultivars Arktis, Novosibirskaya 2 and Ronsard (Table 2) were susceptible only to isolate Tm-258 (resistance group 6), Gourmet and the remaining 24 cultivars were susceptible solely to isolate E (resistance group 5) and Magnifik and MV Zelma were susceptible only to isolate Galina (resistance group 3). These three groups of cultivars can characterise the virulence of the three isolates used.

Cultivars originated from 33 countries, including two from 'pre-1989' countries (Czechoslovakia - six cultivars and the German Democratic Republic-one cultivar, Hadmerslebener Qualitas). European cultivars predominated (410), while there were only 16 cultivars from non-European countries (Argentina, Canada, Japan, Korea, Kazakhstan, Kyrgyzstan and the USA). Cultivars originating from Russia (19) and Turkey (1) were not assigned to either group and the origin of two cultivars (Alomar and Gourmet) is unknown. The most frequent cultivars were from Germany (112), the Czech Republic (104) and France (70).

Cultivars resistant to all three isolates were found in 10 national groups (Table 3), most commonly from the Netherlands (37.5\%), Denmark (33.3\%), Germany (25.5\%), France $(21.5 \%)$, Sweden $(20.0 \%)$ and Great Britain (12.5\%). Such resistance was also found in nine cultivars from three Central European countries (an average of 7.0\%). One (SU-Kae no. 169) out of two cultivars from Korea, and one (Alomar) of two cultivars of unknown origin 
were also resistant to the three isolates. No other cultivar from the remaining 23 countries showed such resistance. Resistance to all three isolates was found in 48 out of 363 (13.2\%) homogeneous European winter cultivars compared with 9 out of 22 (40.9\%) European spring cultivars (both groups differ significantly at $\alpha=0.01$ for binomial distribution).

Table 3. The number of wheat cultivars according to their country of origin and designation as heterogeneous, susceptible or resistant to three powdery mildew isolates.

\begin{tabular}{|c|c|c|c|c|c|c|}
\hline \multirow{2}{*}{$\begin{array}{c}\text { Country } \\
\text { of Origin }{ }^{1}\end{array}$} & \multicolumn{5}{|c|}{ Number of Cultivars } & \multirow{2}{*}{$\begin{array}{c}\text { Resistant } \\
(\%)^{2} \\
\text { (Group 7) }\end{array}$} \\
\hline & Total & Heterogeneous & $\begin{array}{l}\text { Susceptible } \\
\text { (Group 0) }\end{array}$ & $\begin{array}{l}\text { Resistant } \\
\text { (Group 7) }\end{array}$ & $\begin{array}{l}\text { In groups } \\
1-6\end{array}$ & \\
\hline NLD & 9 & 1 & 5 & 3 & 0 & 37.5 \\
\hline DNK & 7 & 1 & 3 & 2 & 1 & 33.3 \\
\hline DEU & 112 & 6 & 70 & 27 & 9 & 25.5 \\
\hline FRA & 70 & 5 & 42 & 14 & 9 & 21.5 \\
\hline SWE & 5 & 0 & 4 & 1 & 0 & 20.0 \\
\hline GBR & 9 & 1 & 6 & 1 & 1 & 12.5 \\
\hline SVK & 9 & 0 & 7 & 1 & 1 & 11.1 \\
\hline AUT & 23 & 0 & 16 & 2 & 5 & 8.7 \\
\hline CZE & 104 & 7 & 75 & 6 & 16 & 6.2 \\
\hline KOR & 2 & 0 & 1 & 1 & 0 & 50.0 \\
\hline Unknown & 2 & 0 & 0 & 1 & 1 & 50.0 \\
\hline ARG & 1 & 0 & 1 & 0 & 0 & 0 \\
\hline BEL & 2 & 0 & 1 & 0 & 1 & 0 \\
\hline BGR & 2 & 0 & 2 & 0 & 0 & 0 \\
\hline $\mathrm{BIH}$ & 1 & 0 & 1 & 0 & 0 & 0 \\
\hline CAN & 1 & 0 & 1 & 0 & 0 & 0 \\
\hline CSK & 6 & 0 & 5 & 0 & 1 & 0 \\
\hline DDR & 1 & 0 & 1 & 0 & 0 & 0 \\
\hline FIN & 2 & 0 & 1 & 0 & 1 & 0 \\
\hline HRV & 4 & 1 & 2 & 0 & 1 & 0 \\
\hline HUN & 11 & 1 & 9 & 0 & 1 & 0 \\
\hline CHE & 8 & 0 & 7 & 0 & 1 & 0 \\
\hline ITA & 2 & 0 & 2 & 0 & 0 & 0 \\
\hline JPN & 3 & 0 & 3 & 0 & 0 & 0 \\
\hline KAZ & 7 & 2 & 5 & 0 & 0 & 0 \\
\hline KGZ & 1 & 0 & 1 & 0 & 0 & 0 \\
\hline MDA & 1 & 0 & 1 & 0 & 0 & 0 \\
\hline POL & 9 & 0 & 9 & 0 & 0 & 0 \\
\hline $\mathrm{ROM}$ & 4 & 0 & 4 & 0 & 0 & 0 \\
\hline RUS & 19 & 2 & 15 & 0 & 2 & 0 \\
\hline SRB & 2 & 0 & 2 & 0 & 0 & 0 \\
\hline TUR & 1 & 0 & 1 & 0 & 0 & 0 \\
\hline UKR & 6 & 1 & 5 & 0 & 0 & 0 \\
\hline USA & 2 & 0 & 2 & 0 & 0 & 0 \\
\hline
\end{tabular}

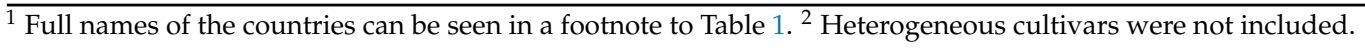

\section{Discussion}

Pathogen resistance can be identified with genetic analyses based on Mendel's laws of inheritance and validated for plant resistance to causal agents of diseases [13], based on a gene-for-gene model [14] using sets of selected pathotypes (resistance gene postulation), or with combinations both these methods [15]. The precondition for the first two methods is genotypic purity of the accession or population after crossing.

Octal notation has been developed [16] and recommended [17] for designating pathotypes (races) of plant pathogens since it clearly concentrates information about their virulence/avirulence patterns. For the same reason it was later adopted for denoting host resistance/susceptibility responses [18] and is now also used here.

Gene bank accessions are commonly characterized by high genotypic heterogeneity [11]. Therefore, ear progenies of the tested cultivars were grown for this project. Despite 
these precautions $28(6.2 \%)$ heterogeneous accessions were revealed possibly resulting from outcrossing during multiplication or mechanical admixtures with other genotypes or during the preparation of accessions. These heterogeneous cultivars could not be assigned to any of the eight resistance groups. A more recent method for identifying resistance in heterogeneous hosts has been developed [15] particularly fit for cereals, which combines genetic analysis and postulation of resistance genes by clusters of selected pathogen isolates. However, this method was not used in the present investigation.

Host-pathogen relationships are binary (resistant or susceptible) and by using three isolates a maximum of eight $\left(2^{3}\right)$ resistance groups could theoretically be identified [16]. The results confirmed the diversity and suitability of the selected $B g t$ isolates because the cultivars could be separated into all eight groups that would theoretically be expected from their phenotypes (resistance responses). This is not surprising because in the Central European population of the "sister" pathogen Bgh the highest biological diversity among all known plant pathogens was found when 226 isolates belonged to 224 pathotypes [5]. With reference to the GWAS project, cultivars from group 0 (no major resistance detected) will be used as preferred recipients of resistances derived from the relatives of bread wheat and also in the search for resistance based on minor genes.

The set of European homogeneous cultivars consisted of two diverse subsets-363 winter and 22 spring wheats. Nevertheless, the proportion of spring wheat resistant to all three isolates $(40.9 \%)$ was more than three times higher than winter wheats $(13.2 \%)$ suggesting that mildew resistance has been a higher priority in breeding spring compared with winter wheat. This is possibly because spring wheat is most vulnerable when its emergence in the field coincides with a high pathogen inoculum spreading from established fields of neighbouring non-resistant winter wheats. This influx of inoculum leads to higher yield losses of spring cultivars and emphasises the importance of resistance breeding programmes.

The analysis of cultivar origin characterized by resistance to all three isolates showed that such resistance was present in 48 out of 203 cultivars of Northwest Europe but in only 9 out of 182 other European wheats (significant difference at $\alpha=0.01$ for binomial distribution). This region has more suitable conditions for pathogen development such as high humidity, milder winters with continuing crop growth and more temperate summers, all of which are conducive for pathogen reproduction and crop damage. To protect wheat from powdery mildew by breeding resistant cultivars must be a priority in the maritime climate of Northwest Europe compared with other regions [19].

Our results show that European bread wheats and mainly those originating from Northwest Europe are rich in major resistances. For example, from 146 Chinese commercial wheat cultivars and breeding lines tested with one Bgt isolate only $15.1 \%$ were resistant, whereas here $23.6 \%$ older accessions were resistant to three isolates. However, $16.4 \%$ of those genotypes showed resistance at the adult-plant stage [20]. These results indicate that other, possibly minor non-specific genes are present in the wheat germplasm.

The public demand for reducing chemical applications and especially those for food production, emphasizes the need for limiting the effects of crop diseases using genetic resistance. According to the adaptability of pathogens [4,21], plant resistance can be divided into two groups [22]. The first group is represented by major genes, which are highly efficient in the absence of virulent pathotypes, but the resistance of most major genes is rapidly overcome by the evolution within the pathogen population [23]. Such resistance, including wheat-powdery mildew pathosystem, is intensively studied and a better understanding could lead to its more widespread use in the breeding of cultivars [24,25]. Another promising way to use major genes is to obtain durable resistance based on loss-of-function mlo gene [26] widely used against barley powdery mildew [27]. Nevertheless, it is unclear how this recessive gene can express the resistance in hexaploid wheat.

The second group of resistances, which includes minor genes, is characterized by lower efficacy because it allows limited reproduction of the pathogen [28,29], but is usually more resistant to pathogen adaptation [30]. Kang et al. [31] summarized details of many resistance genes of both groups, including introgressions from about 30 species of Triticum 
and other relatives and demonstrates the potential of diverse wheat resistance resources to powdery mildew. At the same time, there are plenty of well-characterised low-effect genes in T. aestivum itself [32,33]. These were sufficiently effective in the United Kingdom even when winter wheat had been intensively cultivated under conditions favourable for powdery mildew infection [34] and is appropriate for reducing powdery mildew infection in the field [35]. The results presented in this contribution provide a sound basis for increasing powdery mildew resistance in wheat breeding using the tested cultivars as recipients of novel genes from wheat-related species and/or as a means to accumulate minor resistance genes for improving resistance durability.

\section{Conclusions}

- Breeding and growing resistant cultivars are an environmentally safe and cheap way of disease management.

- In 448 older cultivars from the Czech wheat gene bank, resistance phenotypes against powdery mildew were studied. Despite testing ear progenies 28 accessions were heterogeneous because they were composed of different genotypes.

- In total, 110 cultivars were resistant to one or more of the three isolates that were used, and they could be separated into eight resistance groups.

- Fifty-nine cultivars mostly from Northwest Europe were resistant to all three isolates. The frequency of such cultivars was more than three times higher in spring than in winter wheat accessions. This indicates that more favourable conditions for pathogen development occur in maritime regions and breeding spring wheat with mildew resistance is a priority in these environments.

- In winter wheat, the use of well-characterized low-effect resistance genes (minor genes) against powdery mildew is sufficiently effective.

- The potential of 30 Triticum species and near relatives as valuable resistance sources can be considered.

Funding: This research was funded by the Ministry of Agriculture of the Czech Republic, project no. QK1710302.

Institutional Review Board Statement: Not applicable.

Informed Consent Statement: Not applicable.

Data Availability Statement: All relevant data are presented in this contribution.

Acknowledgments: I would like to thank the Gene Bank and its staff for providing the original seed of wheat cultivars and related information, employees of the breeding company Selgen Ltd. for the propagation and selection of ear progenies, Olga Afanasenko and Miroslav Valárik for providing Bgt isolates, Evsey Kosman for statistical analyses and to Dagmar Krejčířová for perfect preparation of resistance tests.

Conflicts of Interest: Author declare no conflict of interest.

\section{References}

1. Bigini, V.; Camerlengo, F.; Botticella, E.; Sestili, F.; Savatin, D. Biotechnological Resources to Increase Disease-Resistance by Improving Plant Immunity: A Sustainable Approach to Save Cereal Crop Production. Plants 2021, 10, 1146. [CrossRef] [PubMed]

2. Savary, S.; Willocquet, L.; Pethybridge, S.J.; Esker, P.; McRoberts, N.; Nelson, A. The global burden of pathogens and pests on major food crops. Nat. Ecol. Evol. 2019, 3, 430-439. [CrossRef] [PubMed]

3. Dreiseitl, A. Specific Resistance of Barley to Powdery Mildew, Its Use and Beyond. A Concise Critical Review. Genes 2020, 11, 971. [CrossRef] [PubMed]

4. McDonald, B.A.; Linde, C. Pathogen Population Genetics, Evolutionary Potential, and Durable Resistance. Annu. Rev. Phytopathol. 2002, 40, 349-379. [CrossRef]

5. Dreiseitl, A. Great pathotype diversity and reduced virulence complexity in a Central European population of Blumeria graminis f. sp. hordei in 2015-2017. Eur. J. Plant Pathol. 2019, 153, 801-811. [CrossRef]

6. Sánchez-Martín, J.; Keller, B. Contribution of recent technological advances to future resistance breeding. Theor. Appl. Genet. 2019, 132, 713-732. [CrossRef] 
7. Dvorak, J.; Wang, L.; Zhu, T.; Jorgensen, C.M.; Luo, M.-C.; Deal, K.R.; Gu, Y.Q.; Gill, B.S.; Distelfeld, A.; Devos, K.M.; et al. Reassessment of the evolution of wheat chromosomes 4A, 5A, and 7B. Theor. Appl. Genet. 2018, 131, 2451-2462. [CrossRef]

8. Winfield, M.O.; Allen, A.M.; Burridge, A.; Barker, G.L.A.; Benbow, H.R.; Wilkinson, P.A.; Coghill, J.; Waterfall, C.; Davassi, A.; Scopes, G.; et al. High-density SNP genotyping array for hexaploid wheat and its secondary and tertiary gene pool. Plant Biotechnol. J. 2016, 14, 1195-1206. [CrossRef]

9. Janáková, E.; Jakobson, I.; Peusha, H.; Abrouk, M.; Škopová, M.; Šimková, H.; Šafář, J.; Vrána, J.; Doležel, J.; Järve, K.; et al. Divergence between bread wheat and Triticum militinae in the powdery mildew resistance QPm.tut-4A locus and its implications for cloning of the resistance gene. Theor. Appl. Genet. 2018, 132, 1061-1072. [CrossRef]

10. Schmolke, M.; Mohler, V.; Hartl, L.; Zeller, F.J.; Hsam, S.L.K. A new powdery mildew resistance allele at the Pm4 wheat locus transferred from einkorn (Triticum monococcum). Mol. Breed. 2011, 29, 449-456. [CrossRef]

11. Dreiseitl, A. Genotype Heterogeneity in Accessions of a Winter Barley Core Collection Assessed on Postulated Specific Powdery Mildew Resistance Genes. Agronomy 2021, 11, 513. [CrossRef]

12. Torp, J.; Jensen, H.P.; Jørgensen, J.H. Powdery Mildew Resistance Genes in 106 Northwest European Spring Barley Cultivars; Yearbook 1978; Royal Veterinary and Agricultural University: Copenhagen, Denmark, 1978; pp. 75-102.

13. Biffen, R.H. Studies in the inheritance of Disease-Resistance. J. Agric. Sci. 1907, 2, 109-128. [CrossRef]

14. Flor, H.H. Current Status of the Gene-For-Gene Concept. Annu. Rev. Phytopathol. 1971, 9, 275-296. [CrossRef]

15. Dreiseitl, A. A novel way to identify specific powdery mildew resistance genes in hybrid barley cultivars. Sci. Rep. 2020, 10, 18930. [CrossRef]

16. Gilmour, J. Octal Notation for Designating Physiologic Races of Plant Pathogens. Nature 1973, 242, 620. [CrossRef]

17. Limpert, E.; Clifeord, B.; Dreiseitl, A.; Johnson, R.; Müller, K.; Roelfs, A.; Wellings, C. Systems of Designation of Pathotypes of Plant Pathogens. J. Phytopathol. 1994, 140, 359-362. [CrossRef]

18. Dreiseitl, A. Heterogeneity of Powdery Mildew Resistance Revealed in Accessions of the ICARDA Wild Barley Collection. Front. Plant Sci. 2017, 8, 202. [CrossRef]

19. Hsam, S.L.K.; Zeller, F.J. Breeding for powdery mildew resistance in common wheat (Triticum aestivum L.). In The Powdery Mildews: A Comprehensive Treatise; Bélanger, R.R., Bushnell, W.R., Dik, A.J., Carver, T.L.W., Eds.; APS: St. Paul, MN, USA, 2000; pp. 219-238.

20. Ma, K.; Li, X.; Li, Y.; Wang, Z.; Zhao, B.; Wang, B.; Li, Q. Disease Resistance and Genes in 146 Wheat Cultivars (Lines) from the Huang-Huai-Hai Region of China. Agronomy 2021, 11, 1025. [CrossRef]

21. Mundt, C.C. Pyramiding for Resistance Durability: Theory and Practice. Phytopathology 2018, 108, 792-802. [CrossRef]

22. Jørgensen, J.H.; Wolfe, M. Genetics of Powdery Mildew Resistance in Barley. Crit. Rev. Plant Sci. 1994, 13, 97-119. [CrossRef]

23. Poland, J.; Rutkoski, J. Advances and Challenges in Genomic Selection for Disease Resistance. Annu. Rev. Phytopathol. 2016, 54, 79-98. [CrossRef]

24. Müller, M.C.; Praz, C.R.; Sotiropoulos, A.G.; Menardo, F.; Kunz, L.; Schudel, S.; Oberhänsli, S.; Poretti, M.; Wehrli, A.; Bourras, S.; et al. A chromosome-scale genome assembly reveals a highly dynamic effector repertoire of wheat powdery mildew. New Phytol. 2018, 221, 2176-2189. [CrossRef]

25. Bourras, S.; Praz, C.R.; Spanu, P.D.; Keller, B. Cereal powdery mildew effectors: A complex toolbox for an obligate pathogen. Curr. Opin. Microbiol. 2018, 46, 26-33. [CrossRef]

26. Acevedo-Garcia, J.; Spencer, D.; Thieron, H.; Reinstädler, A.; Hammond-Kosack, K.; Phillips, A.L.; Panstruga, R. mlo-based powdery mildew resistance in hexaploid bread wheat generated by a non-transgenic TILLING approach. Plant Biotechnol. J. 2016, 15, 367-378. [CrossRef]

27. Jørgensen, I.H. Discovery, characterization and exploitation of Mlo powdery mildew resistance in barley. Euphytica 1992, 63, 141-152. [CrossRef]

28. Huerta-Espino, J.; Singh, R.; Herrera, L.A.C.; Villaseñor-Mir, H.E.; Rodriguez-Garcia, M.F.; Dreisigacker, S.; Barcenas-Santana, D.; Lagudah, E. Adult Plant Slow Rusting Genes Confer High Levels of Resistance to Rusts in Bread Wheat Cultivars from Mexico. Front. Plant Sci. 2020, 11, 824. [CrossRef]

29. Niks, R.E.; Qi, X.; Marcel, T.C. Quantitative Resistance to Biotrophic Filamentous Plant Pathogens: Concepts, Misconceptions, and Mechanisms. Annu. Rev. Phytopathol. 2015, 53, 445-470. [CrossRef]

30. Cowger, C.; Brown, J.K. Durability of Quantitative Resistance in Crops: Greater Than We Know? Annu. Rev. Phytopathol. 2019, 57, 253-277. [CrossRef]

31. Kang, Y.; Zhou, M.; Merry, A.; Barry, K. Mechanisms of powdery mildew resistance of wheat-A review of molecular breeding. Plant Pathol. 2020, 69, 601-617. [CrossRef]

32. Keller, B.; Wicker, T.; Krattinger, S.G. Advances in Wheat and Pathogen Genomics: Implications for Disease Control. Annu. Rev. Phytopathol. 2018, 56, 67-87. [CrossRef]

33. Krattinger, S.; Keller, B. Molecular genetics and evolution of disease resistance in cereals. New Phytol. 2016, 212, 320-332. [CrossRef] [PubMed]

34. Brown, J.K. Durable Resistance of Crops to Disease: A Darwinian Perspective. Annu. Rev. Phytopathol. 2015, 53, 513-539. [CrossRef] [PubMed]

35. Miedaner, T.; Boeven, A.L.G.-C.; Gaikpa, D.S.; Kistner, M.B.; Grote, C.P. Genomics-Assisted Breeding for Quantitative Disease Resistances in Small-Grain Cereals and Maize. Int. J. Mol. Sci. 2020, 21, 9717. [CrossRef] [PubMed] 